\title{
1 Alternative Splicing Control of Abiotic Stress Responses
}

2 Tom Laloum ${ }^{1}$, Guiomar Martín ${ }^{1}$, and Paula Duque*,

$3 \quad{ }^{1}$ Instituto Gulbenkian de Ciência, 2780-156 Oeiras, Portugal

4 *Correspondence: duquep@igc.gulbenkian.pt

5 Keywords: abiotic stress, abscisic acid (ABA), alternative splicing, posttranscriptional

6 regulation, RNA-binding protein. 


\section{Abstract}

8 Alternative splicing, which generates multiple transcripts from the same gene, is an important

9 modulator of gene expression that can increase proteome diversity and regulate mRNA levels. In plants, this posttranscriptional mechanism is markedly induced in response to environmental stress, with recent studies identifying alternative splicing events that allow quickly adjusting the abundance and function of key stress-response components. In agreement, plant mutants defective in splicing factors are severely impaired in their response to abiotic stress. Notably, mounting evidence indicates that alternative splicing regulates stress responses largely by targeting the abscisic acid (ABA) pathway. We review here the current understanding of posttranscriptional control of plant stress tolerance via alternative splicing and discuss research challenges for the near future.

\section{Pre-mRNA Splicing in Plant Responses to Abiotic Stress}

Under both natural and agricultural contexts, plants are constantly subjected to environmental conditions that adversely affect their growth and development and may threaten their survival. They have therefore evolved a wide spectrum of molecular programs to rapidly perceive changes in the environment and adapt accordingly. The phytohormone abscisic acid (ABA) plays a crucial role in plant responses to major forms of abiotic stress, such as drought, high salinity and extreme temperatures, all of which induce osmotic stress in plant cells. Although osmotic stress also triggers ABA-independent pathways, an adequate plant stress response involves endogenous accumulation of the hormone. Intracellular sensing and signal transduction of $\mathrm{ABA}$ results in the activation of downstream effectors, including transcription factors and ion channels, that implement important adaptive responses to withstand reduced water availability, such as stomatal closure, osmoprotectant synthesis and the induction of a broad range of stress-responsive genes (reviewed in [1,2]).

During the last decade, research has made efficient use of "omics" approaches to identify the transcriptional and translational changes associated with plant perception and responses to abiotic stress (reviewed in [3-6]). While the involvement of posttranscriptional mechanisms is still poorly documented, a new molecular layer regulating these processes has been unfolding. In this review, we discuss emerging evidence that alternative splicing is of central importance to plant abiotic stress tolerance, particularly to ABA-mediated responses, and put forward prospects for future research as well as potential new avenues to improve plant performance under adverse environments.

\section{The Splicing Process}

Precursor-mRNA (pre-mRNA) splicing plays a crucial role in the accurate expression of the information encoded in eukaryotic genomes. This process is carried out by the spliceosome, a large molecular complex that recognizes sequences in the pre-mRNA called splice sites to remove the noncoding introns and join the flanking exons (Figure 1), thus generating a mature transcript. The spliceosome core consists of five small nuclear ribonucleoprotein (snRNPs) and numerous spliceosome-associated proteins, which assemble at introns in a precise order 
(reviewed in [7,8]). Splice site selection is determined not only by core spliceosomal components, but also to a large extent by other RNA-binding proteins, predominantly serine/arginine-rich (SR) proteins and heterogeneous nuclear ribonucleoproteins (hnRNPs), which bind cis-regulatory elements located in either introns or exons, thereby activating or repressing splicing (Figure 1). SR proteins share a multidomain structure characterized by one or two N-terminal RNA Recognition Motifs (RRMs), which bind the RNA targets, and a C-terminal arginine/serine-rich (RS) domain involved in protein-protein interactions that promote recruitment of core spliceosomal components to nearby splice sites. hnRNPs contain a prominent structure of RRMs or K homology RNA-binding domains and auxiliary domains, such as glycine-rich motifs and the arginine-glycine-glycine box, and while frequently described as silencers of splice site selection, their effect appears to depend on the binding position. Both SR proteins (reviewed in [9-11]) and hnRNPs (reviewed in [12-14]) have been implicated in various other steps of posttranscriptional regulation apart from splicing.

Alternative splicing occurs when splice sites are differentially recognized and more than one transcript, and potentially multiple proteins, are generated from the same pre-mRNA. This greatly enhances the coding capacity of a genome, and indeed alternative splicing in humans is known to expand the proteome by at least five times when compared to the number of proteincoding genes [15], while in plants the effects of alternative splicing have not yet been widely addressed at the proteome level. Different types of alternative splicing events can occur in eukaryotes (Box 1), with intron retention being predominant in plants and exon skipping the most frequent alternative splicing event in mammals. Protein isoforms arising from alternative splicing may vary in virtually all aspects of protein function, while specific mRNA splice variants can be targeted for degradation. Possible consequences of alternative splicing at the mRNA and protein levels are detailed in Box 1.

Alternative splicing regulation studies have focused primarily on RNA sequence elements and their associated splicing factors, but extensive work in mammals also points to key roles for chromatin structure, histone modifications and transcription rates (reviewed in [16-18]). Mechanisms of epigenetic control of alternative splicing are emerging in plant systems too ([19]).

\section{Alternative Splicing as a Means to Promote Plant Stress Tolerance}

The current estimate indicates that around $61 \%$ of intron-containing genes in the model plant arabidopsis (Arabidopsis thaliana) undergo alternative splicing [20]. This number is likely to increase as more transcriptome data for plants under different developmental and environmental conditions are evaluated and as more precise tools for identifying splice variants in highthroughput analyses are developed. Notably, RNA-seq data have been confirming previous indications that abiotic stress markedly alters alternative splicing in plants [21-29]. For example, recent studies show that salt stress changes the alternative splicing pattern of more than 6,000 arabidopsis genes [23], while high-temperature stress induces differential splicing of more than 1,000 genes in grape [27]. Moreover, stress imposed by high salinity or treatment with the ABA phytohormone was found to substantially promote the use of non-canonical splice sites $[23,29]$, which could provide a specific means of diversifying the transcriptome in 
response to stress. Although some of these stress-induced changes in plant alternative splicing patterns could result from alterations in other processes, such as turnover and transport of specific splice variants, or reflect a decreased ability of the spliceosome to accurately recognize splice sites under stress conditions, a body of available evidence supports a major role for alternative splicing in implementing adaptive responses to adverse environments. Indeed, plant genes encoding known stress response regulators are particularly prone to generating multiple transcripts via this mechanism [30] and, most importantly, several studies have demonstrated the functional significance of some of these alternative splicing events, which often act as modulators of the ratio between active and non-active isoforms, thereby fine-tuning gene expression in response to abiotic stress.

Tolerance to extreme heat, one of the most detrimental stresses affecting plant productivity, involves evolutionarily conserved heat shock transcription factors (HSFs), which in arabidopsis undergo extensive heat-shock induced alternative splicing events [31]. At least one of these events has been assigned biological impact, as a truncated splice form of HsfA2, but not the full-length isoform, can bind to the HSFA2 promoter and activate its own transcription in a positive auto-regulatory loop [31]. Expression of $H S F$ genes is under the control of the DEHYDRATION-RESPONSIVE ELEMENT BINDING 2 (DREB2) transcription factor, which is also regulated by alternative splicing in response to stress in many plant species [3236]. Indeed, while under normal conditions the $D R E B 2 B$ gene predominantly produces a nonfunctional transcript, the levels of the full-length, functional transcript significantly increase in response to abiotic stress [35,37]. Interestingly, a putative exonic splicing enhancer that affects alternative splicing of the sheepgrass $D R E B 2$ gene was shown to interact in vitro with several SR proteins [36].

High temperatures are also known in plants to enhance endoplasmic reticulum (ER) stress, which triggers the well-conserved unfolded protein response (UPR). ER stress promotes splicing of a small intron in bZIP60, encoding a key transcription factor in UPR, by two ERlocated stress sensors, IRE1a and IRE1b [38,39]. While under normal conditions bZIP60 localizes mostly in the ER membrane, the splice form accumulated during ER stress lacks the transmembrane domain and is therefore targeted to the nucleus [38], where it activates transcription of genes that aid in protein folding and degradation [40,41].

Alternative splicing events in genes encoding downstream effectors of plant stress tolerance have also proved important in regulating stress responses. For example, alternative 3 ' splice site selection gives rise to two splice forms of the arabidopsis Zinc-Induced Facilitator-Like 1 (ZIFL1) transporter - while the full-length isoform is localized in the tonoplast of root cells and regulates transport of the auxin phytohormone, a truncated ZIFL1 variant is targeted to the plasma membrane of leaf stomatal guard cells and mediates drought tolerance [42]. On the other hand, Zinc-Induced Facilitator 2 (ZIF2) is a vacuolar membrane transporter that confers tolerance to zinc by promoting immobilization of the heavy metal in root cells [43]. Remarkably, elevated zinc levels promote an intron retention event in the $5^{\prime}$ untranslated region (5'UTR) of ZIF2 that enhances its own translation [43], thus controlling cellular levels of the encoded protein and thereby plant zinc tolerance. 
It is interesting to note that the vast majority of arabidopsis SR protein genes, which encode key splicing regulators and are extensively alternatively spliced themselves, have their splicing patterns changed by various environmental stresses [21,22,44,45]. Furthermore, Ding et al. [22] found that salt stress induces alternative $5^{\prime}$ and $3^{\prime}$ splice site selection and intron retention events that introduce premature termination codons (PTCs) in SR protein pre-mRNAs, with these changes being accompanied by alterations in the splicing pattern of $49 \%$ of all introncontaining genes. These findings point to an important mode of stress regulation of plant SR proteins, which could function as central coordinators of responses to environmental changes.

\section{Splicing Regulators as Key Mediators of Plant Stress Responses}

In addition to ascribing functional significance to individual alternative splicing events, genetic studies have uncovered major in vivo roles for splicing regulators in plant responses to abiotic stress. This is evidenced by the numerous mutations in spliceosomal components that severely affect plant stress tolerance. Moreover, transcriptome analyses of plants expressing altered levels of splicing regulators have identified potential functional targets of these RNA-binding proteins during the response to stress. Apart from stress-specific regulatory genes, gene ontology enrichment studies of these transcriptomic data have revealed potential alternative splicing feedback loops through the splicing regulation of various other RNA-binding proteins genes $[24,46]$.

Besides the already mentioned posttranscriptional regulation, plant SR protein genes are also regulated by stress at the transcriptional ([45,47,48] and publicly available transcriptomic data) as well as translational levels [27,49], with different environmental cues controlling their phosphorylation status [50,51] and, consequently, subcellular distribution [52-54]. Furthermore, functional evidence is corroborating a role for SR proteins in plant abiotic stress responses. For example, loss of function of the $S R 34 b$ gene, whose expression is upregulated by cadmium (Cd), causes enhanced accumulation of this metal ion and hypersensitivity to its toxicity in arabidopsis [48]. Interestingly, the IRT1 gene, which encodes a putative $\mathrm{Cd}$ transporter, is misspliced in the $s r 34 b$ mutant, providing mechanistic clues on Cd tolerance control by the SR protein [48]. In another study [55], the arabidopsis RS40 and RS41 were found to interact in nuclear speckles with HIGH OSMOTIC STRESS GENE EXPRESSION 5 (HOS5), a KHdomain RNA-binding protein, and FIERY2/CTD phosphatase-like 1 (FRY2/CPL1), a major player in the co-transcriptional processing of nascent transcripts. Knockout mutants for HOS5, RS40 and RS41, all displayed salt and ABA hypersensitivity as well as significant intron retention in many stress-related genes, thus implicating two plant SR proteins in the regulation of alternative splicing under abiotic stress.

Several hnRNPs, particularly glycine-rich RNA-binding proteins (GRPs) whose molecular roles remain largely unknown in plants, have also been functionally implicated in plant abiotic stress responses [56-59]. In arabidopsis, the RZ-1a, a zinc finger-containing GRP, negatively regulates early development under salt and drought stress [56], while heterologous expression of the arabidopsis GRP2 and GRP7 in rice leads to higher grain yields under drought stress [59]. In arabidopsis, GRP7 appears to perform opposite functions in response to different abiotic factors, having a positive effect on stress tolerance under low temperatures and a 
negative effect under salt or dehydration stress conditions, at the seed germination, seedling growth and stomatal movement levels [57]. Notably, using a high-resolution RT-PCR alternative splicing panel and RNA immunoprecipitation (RIP) analyses, Staiger and coworkers [60] showed that GRP7 directly binds mRNA, affecting particularly the choice of alternative 5' splice sites, a function partially shared with its close paralog, GRP8. Future GRP7 studies under abiotic stress conditions should uncover the transcripts targeted by this RNAbinding protein to regulate plant stress tolerance.

The cap-binding proteins CBP20 and CBP80 (also called ABH1 for ABA HYPERSENSITIVE 1) have been reported to modulate plant salt stress responses as well as ABA sensitivity during seed germination and stomatal closure, and to confer drought tolerance [61-64]. These proteins constitute the two subunits of the dimeric nuclear cap-binding complex (CBC), which binds the cap structure of RNA polymerase II transcripts [65] and was found in arabidopsis to influence alternative splicing of first introns, particularly at the 5' splice site [66].

Different arabidopsis spliceosomal components also influence the response to stress in planta. Loss-of-function mutations in the SNW/Ski-interacting protein (SKIP), which physically interacts with the SR-like protein SR45 and functions in alternative splicing through modulation of recognition or cleavage of 5' and 3' splice sites [67], led to plant oversensitivity to salt and osmotic stress [23]. Strikingly, RNA-seq analyses of wild-type and mutant plants under salt stress showed that SKIP controls over $86 \%$ of salt stress-induced alternative splicing events [23]. A component of the U6 snRNP LSm2-8 heptameric complex, LSm5, named SUPERSENSITIVE TO ABSCISIC ACID AND DROUGHT 1 (SAD1) as its loss of function causes exacerbated plant responses to ABA and osmotic stresses [68], also affects the splicing of transcripts associated with high salinity stress [69]. Indeed, overexpression of SAD1 promotes the splicing accuracy and efficiency of stress-responsive genes and improves salt tolerance, while mutations in $S A D 1$ result in a global increase in alternative splicing [69]. It is noteworthy that a protein arginine methyltransferase (PRMT5, also known as SKB1) also affects salt stress-driven pre-mRNA splicing through methylation of another LSm protein, LSm4 [70]. On the other hand, an arabidopsis homolog of the human U5 snRNP-associated $102-\mathrm{kD}$ protein, STABILIZED1 (STA1), is a regulator of plant responses to not only high salinity, but also extreme temperatures and drought stress, controlling the alternative splicing of important stress-induced regulatory genes [71,72]. Finally, RDM16, a U4/U6 snRNP-associated protein as well as a component of the RNA-directed DNA methylation (RdDM) pathway, is required for an appropriate response to salt and ABA stress [73].

It is important to note that, because mRNA splicing is an essential cellular process, the identification of splicing regulators that exert stress-specific functions is rare. In fact, all of the loss-of-function mutants described above (listed in Table 1) show moderate to severe developmental defects, reflecting broader functions for the splicing factors in question. For instance, SKIP antisense transgenic lines show pleiotropic developmental phenotypes, including reduced inflorescence stems and smaller leaves [74], while a recessive STA1 mutant exhibits several defects in leaf and inflorescence morphology [71], and mutations in RDM16 result in dwarf stature as well as smaller leaves and siliques [73]. The identification of RNA-binding protein mutants displaying conditional stress phenotypes would allow the discovery of splicing 
regulators involved specifically in plant responses to abiotic stress. SR proteins appear as

\section{2}

213

214

215

216

217

218

219

220 promising candidates for such a specific role, as several individual knockout mutant lines for these splicing factors are impaired in the response to various stress cues, while showing no evident phenotypes when grown under normal conditions (our unpublished data). Larger and more diverse SR protein families in the plant kingdom (e.g. 18 SR proteins in arabidopsis [75] versus only 12 in humans [76]), resulting from genome amplification [77], could provide a plausible explanation for the occurrence of plant-specific functions, such as in the regulation of stress responses, for a subset of SR protein genes.

\section{An Emerging Link Between Splicing and ABA Signaling}

There is mounting evidence that the ABA phytohormone is a crucial mediator in posttranscriptional regulation of plant stress responses. In fact, most of the splicing regulators described above were also reported to affect ABA sensitivity [55,56,61,62,64,68,70,71,73], suggesting requirement of the hormone for control of abiotic stress responses by these RNAbinding proteins. In addition, the SR-like SR45 protein, which interacts with the U1-70K and $\mathrm{U} 2 \mathrm{AF} 35 \mathrm{~b}$ to facilitate spliceosome assembly at the $5^{\prime}$ and $3^{\prime}$ splice sites [78], regulates sugar responses in arabidopsis by repressing both ABA signaling and glucose-induced accumulation of the hormone [79]. To achieve this, SR45 promotes proteasomal degradation of SnRK1 [46], a protein kinase that coordinates sugar and ABA signaling [80]. Importantly, RNA immunoprecipitation followed by high-throughput sequencing (RIP-seq) revealed that the SR45-bound transcripts are markedly enriched in ABA-signaling functions [81], providing mechanistic insight into the stress roles of SR45.

The involvement of pre-mRNA splicing in ABA stress responses is also corroborated by recent work making use of the splicing inhibitors pladienolide B (PB) and herboxidiene (GEX1a) known in mammalian cells to target the U2 snRNP component SF3b [82,83]. Two studies by Mahfouz and co-authors $[84,85]$ have reported that treatment of arabidopsis plants with either of these compounds partially mimics stress signals, such as high salinity and drought, in a manner reminiscent of ABA, leading to activation of ABA-inducible promoters and stomatal closure. This splicing-dependent activation of stress signaling is substantiated by an increase in intron retention events, also in splice variants previously reported to be pivotal mediators of abiotic stress responses $[85,86]$.

Upon its de novo biosynthesis in response to stress, ABA binds intracellular receptors, inhibiting PP2C phosphatases and thereby derepressing SnRK2 protein kinases [87] that phosphorylate downstream effectors to activate ABA-signaling (Figure 2). Of the few alternative-splicing events in main components of the ABA pathway characterized so far [86,88-90], key relevance in plant adaptation to abiotic stress has been reported for the HAB1 PP2C $[24,86]$, which negatively regulates ABA signaling by binding and dephosphorylating the SnRK2.6 protein kinase [91]. Remarkably, alternative splicing of the arabidopsis $H A B 1$ gene is regulated by ABA and appears to be crucial for switching ABA signal transduction on and off. Although the endogenous relative protein levels of the two HAB1 splice forms were not determined, results reported by Wang et al. [86] are consistent with an accumulation of the fulllength protein under low ABA conditions, which would prevent SnRK2.6 activation, while high 
252 ABA levels would promote retention of a $H A B 1$ intron and result in the production of a

253 truncated splice form that binds but does not inhibit SnRK2.6, thereby activating ABA 254 signaling.

255 ABA-regulated alternative splicing of the HAB1 PP2C is controlled by one of the sole plant 256 RNA-binding proteins reported to fulfill stress-specific roles, RBM25 [24,86]. In fact, while 257 arabidopsis knockout mutants for this human splicing factor homolog are severely affected in 258 ABA, drought and salt stress responses, they display no obvious phenotypes under normal 259 growth conditions [24,92]. Importantly, RBM25 is activated by ABA not only at the 260 transcriptional level, but also posttranslationally through modulation of its phosphorylation 261 levels [24]. Identification of the protein(s) controlling the phosphorylation of RBM25 would 262 shed light on the upstream mechanisms underlying posttranscriptional control of the ABA 263 pathway. Importantly, and consistent with a central role for pre-mRNA splicing in ABA264 mediated stress responses, ABA signaling, SnRK2 kinases in particular, has been shown to 265 regulate the phosphorylation status of several plant splicing factors [50,51,93]. The 266 involvement of splicing regulators (Table 1) and their putative functional targets in ABA 267 responses is summarized in Figure 2.

\section{Concluding Remarks and Future Perspectives}

269 Alternative splicing regulation represents an important means of fine-tuning gene expression 270 that may save the time required for changes in transcriptional activation and pre-mRNA 271 accumulation, thus allowing rapid plant adaptation to adverse environmental conditions.

272 Ultimately, the effects of alternative splicing on mRNAs encoding effectors and modulators of 273 abiotic stress responses are determined by the levels and/or activity of the splicing factors 274 regulating this process. To date, very few studies have addressed the upstream regulatory 275 mechanisms dictating the activity of splicing factors during the response to stress. In mammals, 276 reversible phosphorylation by specific kinases and phosphatases is crucial in the regulation of 277 splicing factor activity [94,95], while in plants the phosphorylation status of several plant SR 278 proteins has been shown to promote their subcellular relocalization to specific nuclear 279 compartments where they are known to control splicing [52-54,96]. Uncovering the upstream 280 components modulating posttranslational modification of plant splicing factors could provide 281 key insight into how environmental signals activate alternative splicing to regulate plant stress 282 tolerance (see Outstanding Questions). Clearly, despite the extensive functional data gathered 283 so far, elucidation of the precise mode of action of splicing regulators controlling plant stress 284 responses will require the identification of their direct mRNA targets. The optimization of state285 of-the-art techniques still challenging in plants, such as individual-nucleotide resolution 286 crosslinking and immunoprecipitation (iCLIP, [97]), should help pinpoint the bona fide 287 physiological targets as well as consensus RNA sequences recognized by these splicing 288 regulators. Remarkably, strong evidence is emerging that ABA signaling is widely regulated at 289 the alternative splicing level. Future identification of new splicing factors and their target 290 mRNAs acting in this pathway should improve our understanding of how alternative splicing 291 modulates plant abiotic stress responses, thus paving the way for new strategies to improve 292 plant productivity under unfavorable environments. 
294

295

296

297

298

299

300

301

302

303

304

305

306

307

308

309

310

311

312

313

314

315

316

317

318

319

320

321

322

323

324

We apologize to the many authors whose work was not cited due to space limitations. T.L. and G.M. are supported by a Marie Skłodowska-Curie Individual Fellowship (MSCA-IF-2015; grant 706274) and an EMBO Long-Term Fellowship (ALTF 1576-2016), respectively. Research in our laboratory is currently funded by Fundação para a Ciência e a Tecnologia (FCT) under grant PTDC/BIA-PLA/1084/2014. Funding from the GREEN-it research unit (UID/Multi/04551/2013) is also acknowledged.

\section{References}

1. Cutler, S.R. et al. (2010) Abscisic acid: emergence of a core signaling network. Annu Rev Plant Biol 61, 651-79

2. Hubbard, K.E. et al. (2010) Early abscisic acid signal transduction mechanisms: newly discovered components and newly emerging questions. Genes Dev 24, 1695-708

3. Imadi, S.R. et al. (2015) Plant transcriptomics and responses to environmental stress: an overview. J Genet 94, 525-37

4. Janmohammadi, M. et al. (2015) Low temperature tolerance in plants: Changes at the protein level. Phytochemistry 117, 76-89

5. Wang, W.Q. et al. (2015) Proteomics of seed development, desiccation tolerance, germination and vigor. Plant Physiol Biochem 86, 1-15

6. Yoshida, T. et al. (2015) Omics Approaches Toward Defining the Comprehensive Abscisic Acid Signaling Network in Plants. Plant Cell Physiol 56, 1043-52

7. Chen, W. and Moore, M.J. (2015) Spliceosomes. Curr Biol 25, R181-3

8. Koncz, C. et al. (2012) The spliceosome-activating complex: molecular mechanisms underlying the function of a pleiotropic regulator. Front Plant Sci 3, 9

9. Long, J.C. and Caceres, J.F. (2009) The SR protein family of splicing factors: master regulators of gene expression. Biochem J 417, 15-27

10. Duque, P. (2011) A role for SR proteins in plant stress responses. Plant Signal Behav 6, 4954

11. Howard, J.M. and Sanford, J.R. (2015) The RNAissance family: SR proteins as multifaceted regulators of gene expression. Wiley Interdiscip Rev RNA 6, 93-110

12. Yeap, W.C. et al. (2014) HnRNP-like proteins as post-transcriptional regulators. Plant Sci 227, 90-100

13. Han, S.P. et al. (2010) Functional diversity of the hnRNPs: past, present and perspectives. Biochem J 430, 379-92

14. Wachter, A. et al. (2012) The Role of Polypyrimidine Tract-Binding Proteins and Other hnRNP Proteins in Plant Splicing Regulation. Front Plant Sci 3, 81

15. Nilsen, T.W. and Graveley, B.R. (2010) Expansion of the eukaryotic proteome by alternative splicing. Nature 463, 457-63

16. Luco, R.F. et al. (2011) Epigenetics in alternative pre-mRNA splicing. Cell 144, 16-26

17. Naftelberg, S. et al. (2015) Regulation of alternative splicing through coupling with transcription and chromatin structure. Annu Rev Biochem 84, 165-98

18. Nieto Moreno, N. et al. (2015) Chromatin, DNA structure and alternative splicing. FEBS Lett 589, 3370-8

19. Pajoro, A. et al. (2017) Histone H3 lysine 36 methylation affects temperature-induced alternative splicing and flowering in plants. Genome Biol 18, 102 
20. Marquez, Y. et al. (2012) Transcriptome survey reveals increased complexity of the alternative splicing landscape in Arabidopsis. Genome Res 22, 1184-95

21. Filichkin, S.A. et al. (2010) Genome-wide mapping of alternative splicing in Arabidopsis thaliana. Genome Res 20, 45-58

22. Ding, F. et al. (2014) Genome-wide analysis of alternative splicing of pre-mRNA under salt stress in Arabidopsis. BMC Genomics 15, 431

23. Feng, J. et al. (2015) SKIP Confers Osmotic Tolerance during Salt Stress by Controlling Alternative Gene Splicing in Arabidopsis. Mol Plant 8, 1038-52

24. Zhan, X. et al. (2015) An Arabidopsis PWI and RRM motif-containing protein is critical for pre-mRNA splicing and ABA responses. Nat Commun 6, 8139

25. Thatcher, S.R. et al. (2016) Genome-Wide Analysis of Alternative Splicing during Development and Drought Stress in Maize. Plant Physiol 170, 586-99

26. Zhang, F. et al. (2016) Genetic regulation of salt stress tolerance revealed by RNA-Seq in cotton diploid wild species, Gossypium davidsonii. Sci Rep 6, 20582

27. Jiang, J. et al. (2017) Integrating Omics and Alternative Splicing Reveals Insights into Grape Response to High Temperature. Plant Physiol 173, 1502-1518

28. Keller, M. et al. (2017) Alternative splicing in tomato pollen in response to heat stress(aEuro). DNA Research 24, 205-217

29. Zhu, F.Y. et al. (2017) Proteogenomic analysis reveals alternative splicing and translation as part of the abscisic acid response in Arabidopsis seedlings. Plant J 91, 518-533

30. Ner-Gaon, H. et al. (2004) Intron retention is a major phenomenon in alternative splicing in Arabidopsis. Plant J39, 877-85

31. Liu, J. et al. (2013) An autoregulatory loop controlling Arabidopsis HsfA2 expression: role of heat shock-induced alternative splicing. Plant Physiol 162, 512-21

32. Xue, G.P. and Loveridge, C.W. (2004) HvDRF1 is involved in abscisic acid-mediated gene regulation in barley and produces two forms of AP2 transcriptional activators, interacting preferably with a CT-rich element. Plant J 37, 326-39

33. Egawa, C. et al. (2006) Differential regulation of transcript accumulation and alternative splicing of a DREB2 homolog under abiotic stress conditions in common wheat. Genes Genet Syst 81, 77-91

34. Qin, F. et al. (2007) Regulation and functional analysis of ZmDREB2A in response to drought and heat stresses in Zea mays L. Plant J 50, 54-69

35. Matsukura, S. et al. (2010) Comprehensive analysis of rice DREB2-type genes that encode transcription factors involved in the expression of abiotic stress-responsive genes. $\mathrm{Mol}$ Genet Genomics 283, 185-96

36. Liu, Z. et al. (2017) Identified of a novel cis-element regulating the alternative splicing of LcDREB2. Sci Rep 7, 46106

37. Peng, X.J. et al. (2013) The transcriptional factor LcDREB2 cooperates with LcSAMDC2 to contribute to salt tolerance in Leymus chinensis. Plant Cell Tissue and Organ Culture $113,245-256$

38. Deng, Y. et al. (2011) Heat induces the splicing by IRE1 of a mRNA encoding a transcription factor involved in the unfolded protein response in Arabidopsis. Proc Natl Acad Sci U S A 108, 7247-52

39. Nagashima, Y. et al. (2011) Arabidopsis IRE1 catalyses unconventional splicing of bZIP60 mRNA to produce the active transcription factor. Sci Rep 1, 29

40. Iwata, Y. et al. (2008) Arabidopsis bZIP60 is a proteolysis-activated transcription factor involved in the endoplasmic reticulum stress response. Plant Cell 20, 3107-21 
41. Wang, B. et al. (2017) BhbZIP60 from Resurrection Plant Boea hygrometrica Is an mRNA Splicing-Activated Endoplasmic Reticulum Stress Regulator Involved in Drought Tolerance. Front Plant Sci 8, 245

42. Remy, E. et al. (2013) A major facilitator superfamily transporter plays a dual role in polar auxin transport and drought stress tolerance in Arabidopsis. Plant Cell 25, 901-26

43. Remy, E. et al. (2014) Intron retention in the 5'UTR of the novel ZIF2 transporter enhances translation to promote zinc tolerance in arabidopsis. PLoS Genet 10, e1004375

44. Palusa, S.G. et al. (2007) Alternative splicing of pre-mRNAs of Arabidopsis serine/arginine-rich proteins: regulation by hormones and stresses. Plant J 49, 1091-107

45. Tanabe, N. et al. (2007) Differential expression of alternatively spliced mRNAs of Arabidopsis SR protein homologs, atSR30 and atSR45a, in response to environmental stress. Plant Cell Physiol 48, 1036-49

46. Carvalho, R.F. et al. (2016) The Arabidopsis SR45 Splicing Factor, a Negative Regulator of Sugar Signaling, Modulates SNF1-Related Protein Kinase 1 Stability. Plant Cell 28, 191025

47. Cruz, T.M. et al. (2014) Abscisic acid (ABA) regulation of Arabidopsis SR protein gene expression. Int J Mol Sci 15, 17541-64

48. Zhang, W. et al. (2014) Splicing factor SR34b mutation reduces cadmium tolerance in Arabidopsis by regulating iron-regulated transporter 1 gene. Biochem Biophys Res Commun 455, 312-7

49. Palusa, S.G. and Reddy, A.S. (2015) Differential recruitment of splice variants from SR pre-mRNAs to polysomes during development and in response to stresses. Plant Cell Physiol 56, 421-7

50. Umezawa, T. et al. (2013) Genetics and phosphoproteomics reveal a protein phosphorylation network in the abscisic acid signaling pathway in Arabidopsis thaliana. Sci Signal 6, rs8

51. Wang, P. et al. (2013) Quantitative phosphoproteomics identifies SnRK2 protein kinase substrates and reveals the effectors of abscisic acid action. Proc Natl Acad Sci U S A 110, 11205-10

52. Ali, G.S. et al. (2003) Nuclear localization and in vivo dynamics of a plant-specific serine/arginine-rich protein. Plant J 36, 883-93

53. Tillemans, V. et al. (2006) Insights into nuclear organization in plants as revealed by the dynamic distribution of Arabidopsis SR splicing factors. Plant Cell 18, 3218-34

54. Rausin, G. et al. (2010) Dynamic nucleocytoplasmic shuttling of an Arabidopsis SR splicing factor: role of the RNA-binding domains. Plant Physiol 153, 273-84

55. Chen, T. et al. (2013) A KH-domain RNA-binding protein interacts with FIERY2/CTD phosphatase-like 1 and splicing factors and is important for pre-mRNA splicing in Arabidopsis. PLoS Genet 9, e1003875

56. Kim, Y.O. et al. (2007) A zinc finger-containing glycine-rich RNA-binding protein, atRZ1a, has a negative impact on seed germination and seedling growth of Arabidopsis thaliana under salt or drought stress conditions. Plant Cell Physiol 48, 1170-81

57. Kim, J.S. et al. (2008) Glycine-rich RNA-binding protein 7 affects abiotic stress responses by regulating stomata opening and closing in Arabidopsis thaliana. Plant J 55, 455-66

58. Yeap, W.C. et al. (2012) EgRBP42 encoding an hnRNP-like RNA-binding protein from Elaeis guineensis Jacq. is responsive to abiotic stresses. Plant Cell Rep 31, 1829-43

59. Yang, D.H. et al. (2014) Expression of Arabidopsis glycine-rich RNA-binding protein AtGRP2 or AtGRP7 improves grain yield of rice (Oryza sativa) under drought stress conditions. Plant Sci 214, 106-12 
60. Streitner, C. et al. (2012) An hnRNP-like RNA-binding protein affects alternative splicing by in vivo interaction with transcripts in Arabidopsis thaliana. Nucleic Acids Res 40, $11240-55$

61. Hugouvieux, V. et al. (2001) An mRNA cap binding protein, ABH1, modulates early abscisic acid signal transduction in Arabidopsis. Cell 106, 477-87

62. Papp, I. et al. (2004) A mutation in the Cap Binding Protein 20 gene confers drought tolerance to Arabidopsis. Plant Mol Biol 55, 679-86

63. Kong, X. et al. (2014) Quantitative proteomics analysis reveals that the nuclear cap-binding complex proteins arabidopsis CBP20 and CBP80 modulate the salt stress response. $J$ Proteome Res 13, 2495-510

64. Daszkowska-Golec, A. et al. (2017) Mutation in HvCBP20 (Cap Binding Protein 20) Adapts Barley to Drought Stress at Phenotypic and Transcriptomic Levels. Front Plant Sci 8,942

65. Izaurralde, E. et al. (1994) A nuclear cap binding protein complex involved in pre-mRNA splicing. Cell 78, 657-68

66. Raczynska, K.D. et al. (2010) Involvement of the nuclear cap-binding protein complex in alternative splicing in Arabidopsis thaliana. Nucleic Acids Res 38, 265-78

67. Wang, X. et al. (2012) SKIP is a component of the spliceosome linking alternative splicing and the circadian clock in Arabidopsis. Plant Cell 24, 3278-95

68. Xiong, L. et al. (2001) Modulation of abscisic acid signal transduction and biosynthesis by an Sm-like protein in Arabidopsis. Dev Cell 1, 771-81

69. Cui, P. et al. (2014) Dynamic regulation of genome-wide pre-mRNA splicing and stress tolerance by the Sm-like protein LSm5 in Arabidopsis. Genome Biol 15, R1

70. Zhang, Z. et al. (2011) Arabidopsis floral initiator SKB1 confers high salt tolerance by regulating transcription and pre-mRNA splicing through altering histone H4R3 and small nuclear ribonucleoprotein LSM4 methylation. Plant Cell 23, 396-411

71. Lee, B.H. et al. (2006) STABILIZED1, a stress-upregulated nuclear protein, is required for pre-mRNA splicing, mRNA turnover, and stress tolerance in Arabidopsis. Plant Cell 18, $1736-49$

72. Kim, G.D. et al. (2017) STABILIZED1 Modulates Pre-mRNA Splicing for Thermotolerance. Plant Physiol 173, 2370-2382

73. Huang, C.F. et al. (2013) A Pre-mRNA-splicing factor is required for RNA-directed DNA methylation in Arabidopsis. PLoS Genet 9, e1003779

74. Lim, G.H. et al. (2010) A putative novel transcription factor, AtSKIP, is involved in abscisic acid signalling and confers salt and osmotic tolerance in Arabidopsis. New Phytol $185,103-13$

75. Barta, A. et al. (2010) Implementing a rational and consistent nomenclature for serine/arginine-rich protein splicing factors (SR proteins) in plants. Plant Cell 22, 2926-9

76. Manley, J.L. and Krainer, A.R. (2010) A rational nomenclature for serine/arginine-rich protein splicing factors (SR proteins). Genes Dev 24, 1073-4

77. Kalyna, M. and Barta, A. (2004) A plethora of plant serine/arginine-rich proteins: redundancy or evolution of novel gene functions? Biochem Soc Trans 32, 561-4

78. Day, I.S. et al. (2012) Interactions of SR45, an SR-like protein, with spliceosomal proteins and an intronic sequence: insights into regulated splicing. Plant $J 71,936-47$

79. Carvalho, R.F. et al. (2010) The plant-specific SR45 protein negatively regulates glucose and ABA signaling during early seedling development in Arabidopsis. Plant Physiol 154, $772-83$ 
80. Rodrigues, A. et al. (2013) ABI1 and PP2CA phosphatases are negative regulators of Snf1related protein kinase1 signaling in Arabidopsis. Plant Cell 25, 3871-84

81. Xing, D. et al. (2015) Transcriptome-Wide Identification of RNA Targets of Arabidopsis SERINE/ARGININE-RICH45 Uncovers the Unexpected Roles of This RNA Binding Protein in RNA Processing. Plant Cell 27, 3294-308

82. Kotake, Y. et al. (2007) Splicing factor SF3b as a target of the antitumor natural product pladienolide. Nat Chem Biol 3, 570-5

83. Hasegawa, M. et al. (2011) Identification of SAP155 as the target of GEX1A (Herboxidiene), an antitumor natural product. ACS Chem Biol 6, 229-33

84. AlShareef, S. et al. (2017) Herboxidiene triggers splicing repression and abiotic stress responses in plants. BMC Genomics 18, 260

85. Ling, Y. et al. (2017) Pre-mRNA splicing repression triggers abiotic stress signaling in plants. Plant $J$ 89, 291-309

86. Wang, Z. et al. (2015) ABA signalling is fine-tuned by antagonistic HAB1 variants. Nat Commun 6, 8138

87. Umezawa, T. et al. (2009) Type 2C protein phosphatases directly regulate abscisic acidactivated protein kinases in Arabidopsis. Proc Natl Acad Sci U S A 106, 17588-93

88. Sugliani, M. et al. (2010) The conserved splicing factor SUA controls alternative splicing of the developmental regulator ABI3 in Arabidopsis. Plant Cell 22, 1936-46

89. Gao, Y. et al. (2013) Functional characterization of two alternatively spliced transcripts of tomato ABSCISIC ACID INSENSITIVE3 (ABI3) gene. Plant Mol Biol 82, 131-45

90. Wang, M. et al. (2013) BolOST1, an ortholog of Open Stomata 1 with alternative splicing products in Brassica oleracea, positively modulates drought responses in plants. Biochem Biophys Res Commun 442, 214-20

91. Vlad, F. et al. (2009) Protein phosphatases 2C regulate the activation of the Snf1-related kinase OST1 by abscisic acid in Arabidopsis. Plant Cell 21, 3170-84

92. Cheng, C. et al. (2017) RBM25 Mediates Abiotic Responses in Plants. Front Plant Sci 8, 292

93. Li, J. et al. (2002) Modulation of an RNA-binding protein by abscisic-acid-activated protein kinase. Nature 418, 793-7

94. Stamm, S. (2008) Regulation of alternative splicing by reversible protein phosphorylation. $J$ Biol Chem 283, 1223-7

95. Zhou, Z. and Fu, X.D. (2013) Regulation of splicing by SR proteins and SR protein-specific kinases. Chromosoma 122, 191-207

96. Stankovic, N. et al. (2016) Dynamic Distribution and Interaction of the Arabidopsis SRSF1 Subfamily Splicing Factors. Plant Physiol 170, 1000-13

97. Huppertz, I. et al. (2014) iCLIP: protein-RNA interactions at nucleotide resolution. Methods $65,274-87$

\section{Figure Legends and Boxes}

\section{Box 1. Alternative Splicing: Different Types and Molecular Outcomes}

Splicing of a nascent pre-mRNA occurs in the nucleus, mostly cotranscriptionally, when the spliceosome recognizes nucleotide sequences defining exon/intron boundaries called splice sites to remove the introns, join the exons, and generate a mature mRNA molecule. Importantly, detection of splice sites by the spliceosome can vary, thereby producing alternative mature transcripts from the same pre-mRNA. This mechanism, termed alternative splicing, can 
change the structure of transcripts in different ways and have multiple molecular consequences. For example, intron retention occurs when both the 5' and 3' ends of a specific intron are not recognized, splicing fails, and a mature mRNA that includes the intronic sequence is produced (see Figure I). Also, depending on the correct recognition of either or both splice sites flanking an exon, the latter can fail to be included in the final transcript, and selection of alternative 5' or 3' splice sites results in the inclusion of different stretches of an exon. The insertion or deletion of alternative fragments in an mRNA can generate proteins with altered domains, and thereby affect key aspects of their molecular function, such as enzymatic activity, subcellular localization, stability, binding properties or posttranslational modifications. By contrast, when the alternatively-spliced sequence affects noncoding regions such as untranslated regions (UTRs) or introns, the stability, nuclear export, localization and/or translation of the mRNA may be affected. Importantly, when alternative splicing causes a change in the open reading frame (ORF), a premature termination codon (PTC) is often introduced in the mRNA. Specific proteins in the cell can then recognize this PTC during the first round of translation and target the transcript for degradation through a cytoplasmic mRNA surveillance system known as nonsense-mediated decay (NMD).

\section{Figure I. Schematic Representation of Different Alternative Splicing Types}

Figure 1. Schematic Representation of the Splicing Process. Canonical sequences in the precursor mRNA (pre-mRNA) define the splice sites $\left(5^{\prime}\right.$ splice site, branch point, polypyrimidine tract, and $3^{\prime}$ splice site), while additional cis-regulatory elements in introns (ISRs) or exons (ESRs) modulate the recognition of splice sites by the spliceosome. This multimegadalton complex is composed of five snRNPs, named U1, U2, U4, U5 and U6, and a range of spliceosome-associated non-snRNP proteins, such as the U2 auxiliary factor (U2AF), required for the binding of snRNPs to the pre-mRNA. In addition, SR proteins and hnRNPs regulate the efficiency of splice site recognition, with the former generally binding ESRs to enhance splicing and the latter antagonizing this effect by binding ISRs and repressing splicing, though several exceptions to these effects have been reported. Variations in the selection of splice sites result in the production of different mRNA molecules from the same pre-mRNA through alternative splicing - in the example shown, an hnRNP recognizing an intronic silencer sequence inhibits splicing of an intron, leading to its retention in one of the two alternative transcripts produced. Abbreviations: 5' SS, 5' splice site; BP, branch point; $(\mathrm{Y})_{\mathrm{n}}$, polypyrimidine tract; 3' SS, 3' splice site; ISR, intronic splicing regulator; ESR, exonic splicing regulator; snRNP, small nuclear ribonucleoprotein; SR, serine/arginine-rich (SR) protein; hnRNP, heterogeneous ribonucleoprotein.

Figure 2. Interplay Between Alternative Splicing and ABA Signaling. ABA is a major mediator of plant responses to abiotic stress. Drought, high salinity and extreme temperatures all induce osmotic stress in plant cells, triggering ABA biosynthesis. Binding of the hormone to its intracellular receptors inhibits PP2C phosphatases, thereby derepressing SnRK2 protein kinases that through phosphorylation activate proteins with different molecular functions, thus implementing the diverse physiological and developmental responses that allow plants to withstand environmental stress. An increasing number of splicing factors is being reported to bind and regulate the processing of mRNAs encoding ABA signaling components. The bestcharacterized example of a single alternative splicing event impacting ABA physiological 
responses is that of the RBM25 splicing factor [24,86], which under abiotic stress is activated

568 by ABA and binds the $H A B 1$ PP2C pre-mRNA to ensure it is spliced correctly and generates a 569 functional protein. In the absence of ABA and therefore RBM25 activation, the last intron of $570 H A B 1$ is retained, producing a non-functional protein that is unable to transduce the stress 571 signal via the ABA pathway. Finally, increased complexity in the interplay between ABA 572 signaling (shown in green) and pre-mRNA splicing (shown in pink) is underscored by evidence 573 that ABA or related stresses can regulate splicing factor levels and/or activity. This regulation 574 includes transcriptional, posttranscriptional and posttranslational mechanisms. Interestingly, 575 some data also suggest that central players in the ABA signaling pathway could directly affect 576 the activity of splicing factors by regulating their phosphorylation status [50,51]. Abbreviations: 577 SF, splicing factor; TF, transcription factor.

\section{Table 1. Splicing Factors Involved In Plant Abiotic Stress Responses}

\begin{tabular}{|c|c|c|c|c|c|c|c|c|}
\hline \multirow{2}{*}{\multicolumn{2}{|c|}{ Splicing factor }} & \multicolumn{6}{|c|}{ Abiotic stress under which in vivo role was reported } & \multirow{3}{*}{$\begin{array}{c}\text { References } \\
{[46,81]} \\
\end{array}$} \\
\hline & & \multirow{2}{*}{$\frac{\mathrm{ABA}}{\checkmark}$} & \multirow{2}{*}{$\frac{\text { drought }}{x}$} & \multirow{2}{*}{$\begin{array}{c}\text { salt } \\
x\end{array}$} & \multirow{2}{*}{$\begin{array}{c}\text { cold } \\
x\end{array}$} & \multirow{2}{*}{$\frac{\text { heat }}{x}$} & \multirow{2}{*}{$\frac{\text { cadmium }}{x}$} & \\
\hline \multirow{4}{*}{ 金 } & SR45 & & & & & & & \\
\hline & SR34b & $x$ & $x$ & $x$ & $x$ & $x$ & $\checkmark$ & [48] \\
\hline & RS40 & $\checkmark$ & $x$ & $\checkmark$ & $x$ & $x$ & $x$ & [55] \\
\hline & RS41 & $\checkmark$ & $x$ & $\checkmark$ & $x$ & $x$ & $x$ & {$[55]$} \\
\hline \multirow{3}{*}{$\frac{n}{0}$} & GRP2 & $x$ & $\checkmark$ & $x$ & $x$ & $x$ & $x$ & {$[59]$} \\
\hline & GRP7 & $x$ & $\checkmark$ & $\checkmark$ & $\checkmark$ & $x$ & $x$ & {$[57,59]$} \\
\hline & RZ-1a & $\checkmark$ & $\checkmark$ & $\checkmark$ & $x$ & $x$ & $x$ & {$[56]$} \\
\hline \multirow{2}{*}{$\stackrel{n}{0}$} & CBP20 & $\checkmark$ & $\checkmark$ & $\checkmark$ & $x$ & $x$ & $x$ & [62-64] \\
\hline & СВР80/ABH1 & $\checkmark$ & $\checkmark$ & $\checkmark$ & $x$ & $x$ & $x$ & {$[61,63]$} \\
\hline \multirow{6}{*}{ 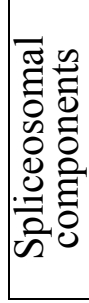 } & SKIP & $x$ & $\checkmark$ & $\checkmark$ & $x$ & $x$ & $x$ & {$[23]$} \\
\hline & SAD1 & $\checkmark$ & $\checkmark$ & $\checkmark$ & $x$ & $x$ & $x$ & {$[68,69]$} \\
\hline & LSm4 & $\checkmark$ & $x$ & $\checkmark$ & $x$ & $x$ & $x$ & [70] \\
\hline & RDM16 & $\checkmark$ & $x$ & $\checkmark$ & $x$ & $x$ & $x$ & [73] \\
\hline & STA1 & $\checkmark$ & $\checkmark$ & $\checkmark$ & $\checkmark$ & $\checkmark$ & $x$ & [71] \\
\hline & RBM25 & $\checkmark$ & $\checkmark$ & $\checkmark$ & $x$ & $x$ & $x$ & {$[24,86,92]$} \\
\hline
\end{tabular}

\section{Glossary}

580 Abiotic stress: detrimental effect of environmental (nonliving) factors - e.g. extreme 581 temperatures, drought, flooding, toxic compounds — on living organisms such as plants.

582 Abscisic acid (ABA): an isoprenoid plant hormone involved in various developmental 583 processes - e.g. seed maturation and germination, seed and bud dormancy, floral transition 584 and a major player in mediating plant responses to abiotic stress through regulation of stomatal 585 closure and induction of the expression of stress response genes.

586 Alternative splicing: occurs when splice sites are differentially recognized and multiple 587 transcripts are generated from the same pre-mRNA, greatly enhancing the coding capacity of 588 the genome and providing a means of regulating gene expression.

589 Heterogeneous nuclear ribonucleoproteins (hnRNPs): a large family of structurally diverse 590 RNA-binding proteins, usually consisting of several RNA-binding domains connected by linker 
591 regions of varying length, involved in multiple aspects of nucleic acid metabolism, such as

592 alternative splicing, mRNA stability, or transcriptional and translational regulation.

593 Isoform: a version of a protein showing a similar but not identical amino acid sequence that, 594 when originating from the same pre-mRNA, often results from alternative splicing.

595 Precursor-mRNA (pre-mRNA): a single strand of messenger RNA (mRNA), produced by 596 transcription of the genomic DNA, that has yet to be processed or has been processed 597 incompletely.

598 Pre-mRNA Splicing: the stepwise process by which introns are excised from the pre-mRNA 599 and the exons joined to produce a mature mRNA molecule.

600 Serine/arginine-rich (SR) proteins: a conserved family of RNA-binding proteins involved 601 mainly in pre-mRNA splicing - but also implicated in other posttranscriptional functions, such 602 as mRNA export, stability or translation - characterized by the presence of one or two N603 terminal RNA-recognition motifs (RRMs) and a C-terminal arginine/serine dipeptide-rich RS 604 domain involved in protein interactions.

605 small nuclear ribonucleoproteins (snRNPs): RNA-protein complexes comprising small 606 nuclear RNAs (snRNAs) and many nuclear proteins - the five snRNPs that form the 607 spliceosome, called U1, U2, U4, U5, and U6, are all essential for the removal of introns from 608 pre-mRNAs.

609 Spliceosome: a large and complex molecular apparatus, composed of five snRNPs and 610 numerous spliceosome-associated proteins, that carries out the splicing reaction.

611 Osmotic stress: negative impact of a sudden change in solute concentration, causing a rapid 612 passage of water or another solvent across a membrane by osmosis, which in living cells can 613 result in cell lysis (rupture of the plasma membrane). 


\section{Trends}

2 Alternative splicing, which generates multiple transcripts and potentially more than one 3 protein from the same gene, is markedly changed by environmental stresses that 4 negatively impact plant growth and development.

5 Plant stress-related genes are particularly prone to alternative splicing events, which often

6 modulate the ratio between active and non-active isoforms in response to abiotic stress,

7 thus fine-tuning the expression of key stress regulators.

8 Recent genetic and transcriptomic analyses identified important roles for numerous 9 splicing factors in the control of plant abiotic stress responses.

10 Emerging evidence indicates that splicing factors modulate stress responses by targeting 11 components of the ABA pathway, unveiling a novel regulatory layer in plant stress 12 tolerance. 


\section{Outstanding Questions}

2 Which are the bona fide physiological targets of splicing factors under abiotic stress?

3 And which RNA consensus sequences do they bind? Though challenging, the

4 optimization of techniques such as CLIP or improvements of this protocol in plant

5 systems holds much promise for the identification of the binding motifs and mRNAs

6 targeted directly by splicing factors to regulate plant abiotic stress tolerance.

7 How do splicing factors integrate environmental signals to regulate alternative splicing?

8 While large-scale transcriptomic data revealing marked splicing changes in response to

9 stress are accumulating at a fast pace, the upstream regulatory mechanisms dictating the

10 activity of splicing regulators under stress conditions remain elusive. Unraveling these

11 mechanisms will require in-depth studies of posttranslational modifications, particularly

12 phosphorylation, and of the splicing factor protein interactors essential to their function.

13 How and to what extent does alternative splicing control ABA signaling during the

14 response to abiotic stress? What are the components of the ABA pathway targeted by

15 splicing regulators? Is the activity of these splicing factors controlled in an ABA-

16 dependent manner? Answering these questions will provide crucial insight into how

17 alternative splicing modulates plant stress responses and contribute new approaches to

18 improve crop tolerance under environmental stress. 


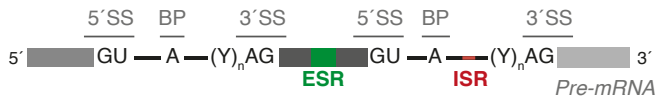
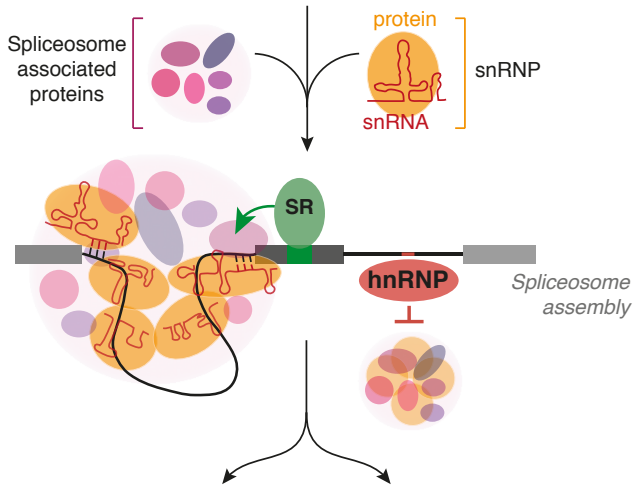
Constitutive splicing

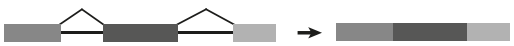

Intron retention

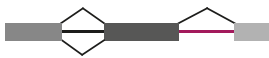

Exon skipping

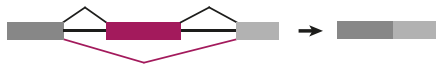

Alternative 5'splice sites

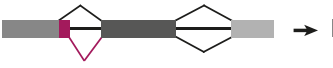

Alternative 3'splice sites
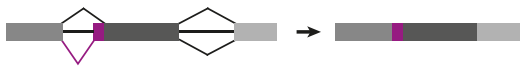


\section{Salt Heat}

\section{Drought $\downarrow \downarrow$ Cold \\ osmotic \\ sensor}

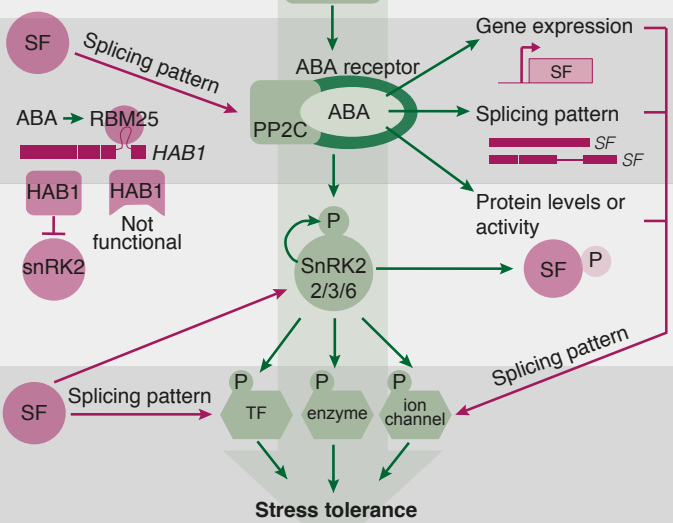

